\title{
Exacerbation of Ischemic Dysfunction by Angiotensin II in Red Cell-perfused Rabbit Hearts Effects on Coronary Flow, Contractility, and High-Energy Phosphate Metabolism
}

Takatoshi Mochizuki, “ Franz R. Eberli, ${ }^{\circ}$ Carl S. Apstein, ${ }^{\star}$ and Beverly H. Lorell*

*Charles A. Dana Research Institute and Harvard-Thorndike Laboratory of Beth Israel Hospital, and Cardiovascular Division, Department of Medicine, Beth Israel Hospital and Harvard Medical School, Boston, Massachusetts 02215; and ${ }^{\ddagger}$ Cardiac Muscle Research Laboratory, Whitaker Cardiovascular Institute, Boston University School of Medicine, and the Boston City Hospital, Boston, Massachusetts 02118

\begin{abstract}
We studied the effects of angiotensin II during low-flow ischemia and reperfusion using red cell-perfused isovolumic rabbit hearts. Under baseline conditions where coronary perfusion pressure (CPP) was $100 \mathrm{~mm} \mathrm{Hg}$ and left ventricular end-diastolic pressure (LVEDP) was set at $10 \mathrm{~mm} \mathrm{Hg}, 10^{-8} \mathrm{M}$ angiotensin II caused a mild increase in $\mathrm{LV}$ developed pressure $(+12 \%)$ and decrease in coronary flow $(-8 \%)$. Low-flow ischemia was imposed by reducing CPP to $15 \mathrm{~mm} \mathrm{Hg}$ for $30 \mathrm{~min}$ followed by $30 \mathrm{~min}$ of reperfusion. During ischemia, the angiotensin II group showed a gradual further reduction in coronary flow in association with a greater depression of $\mathrm{LV}$ developed pressure and increase in LVEDP relative to the no-drug group. To separate the effect of angiotensin II on coronary flow from a direct myocardial effect, the angiotensin II group was compared with an additional no-drug group with a matched progressive reduction in coronary flow during ischemia. In these groups, the ischemic depression of $\mathrm{LV}$ developed pressure, myocardial ATP levels, and lactate production were similar. However, the ischemic rise in LVEDP was greater $(42.0 \pm 5.4$ vs. $19.9 \pm 1.3 \mathrm{~mm}$ $\mathrm{Hg}, P<0.01)$ and recovery was incomplete in the angiotensin II group. These observations suggest that angiotensin II exerts a direct adverse effect on $\mathrm{LV}$ diastolic relaxation during low-flow ischemia and recovery. (J. Clin. Invest. 1992. 89:490-498.) Key words: adenosine triphosphate • angiotensin II • diastole • ischemia
\end{abstract}

\section{Introduction}

Transient myocardial ischemia in patients with coronary artery disease is associated with a severe and reversible depression of myocardial relaxation and elevation of left ventricular enddiastolic pressure (LVEDP) (1-3). ${ }^{1}$ We have previously shown that these acute and reversible changes in diastolic function can

Address reprint requests to Dr. Lorell, Cardiovascular Division, Beth Israel Hospital, 330 Brookline Avenue, Boston, MA 02215.

Received for publication 29 May 1991 and in revised form $16 \mathrm{Au}$ gust 1991.

1. Abbreviations used in this paper: $\mathrm{ACE}$, angiotensin-converting enzyme; CP, creatine phosphate; CPP, coronary perfusion pressure; LVEDP, left ventricular end-diastolic pressure.

J. Clin. Invest.

(C) The American Society for Clinical Investigation, Inc. 0021-9738/92/02/0490/09 \$2.00

Volume 89, February 1992, 490-498 be simulated in isolated red cell-perfused hearts in which the critical feature is that ischemia is imposed by imposition of a preserved but greatly reduced level of coronary flow, sufficient to sustain systolic force development, in contrast with primary no-flow ischemia which results in immediate cessation of contractile function, loss of all coronary turgor, and an early downward shift in the LV diastolic pressure-volume relation (4-6). These acute and reversible changes in relaxation and diastolic function during low-flow ischemia and during hypoxia are postulated to be related in part to myocardial ATP depletion in association with the reversible elevation of cytosolic $\mathrm{Ca}^{2+}(7,8)$. Consistent with these postulated mechanisms, the rapid and reversible changes in relaxation and diastolic distensibility that occur during low-flow ischemia are exacerbated by interventions which increase $\mathrm{Ca}^{2+}$ availability and myocardial energy demand $(4,5)$ and are more pronounced in hypertrophied hearts with intrinsic impairment of cytosolic $\mathrm{Ca}^{2+}$ handling (9-11).

However, the potential contribution of the renin-angiotensin system to the physiologic and metabolic response of the heart to low-flow ischemia has received little attention. In this regard, there is substantial evidence which supports the presence of an endogenous renin-angiotensin system in the heart (12-14). Experiments in isolated perfused hearts by Lindpaintner et al. (15) and from our laboratories (16) have demonstrated the intracardiac activation of angiotensin I to II, and we have recently shown that angiotensin-converting enzyme (ACE) mRNA is expressed in the heart (16). Under well-oxygenated conditions, the physiologic action of angiotensin II on the heart may include local effects on cardiac contractility and coronary vasomotor tone as well as a role in modulating cell growth $(12,15-17)$. However, there is indirect evidence that the availability of angiotensin II to the heart during ischemia may be deleterious. Recent studies have suggested that specific inhibition of angiotensin II activation has the potential to protect against ischemia and reperfusion injury (18-20). However, the effects of angiotensin II on myocardial contractility and relaxation, coronary vasomotor tone, and metabolism during low-flow ischemia are not known.

To test the hypotheses that angiotensin II directly impairs the preservation of contractile function during low-flow ischemia and exacerbates myocardial stunning during reperfusion, we compared the effects of $30 \mathrm{~min}$ of low-flow ischemia and reperfusion on coronary vasoreactivity, systolic and diastolic function, and myocardial lactate production in isolated isovolumic red cell-perfused rabbit hearts perfused with $10^{-8} \mathrm{M}$ angiotensin II in comparison with control hearts without drug. Myocardial ATP and creatine phosphate levels were measured in additional hearts at end-ischemia. Our experiments demon- 
strated that angiotensin II directly impaired LV ventricular diastolic relaxation during ischemia and its recovery independent of its effects on coronary vascular tone and systolic function or the magnitude of high energy phosphate depletion.

\section{Methods}

Experimental preparation. We used an isolated isovolumic red cellperfused rabbit heart preparation that has been developed in our laboratory and has been described previously in detail (4-6). Male, albino New Zealand rabbits (1.3-2.2 kg) were heparinized (1,000 U, intravenously) and anesthetized with sodium pentobarbital $(50 \mathrm{mg} / \mathrm{kg}$, intravenously). After thoracotomy, the heart was isolated, a short perfusion cannula was inserted into the ascending aortic stump and positioned immediately above the aortic valve, and the coronary arteries were perfused via the aortic root with the red cell-containing perfusate. The interval between isolation of the heart and initiation of coronary perfusion in all experiments was $<15 \mathrm{~s}$.

A bovine red cell perfusate was used which was developed by Marshall and Zhang (21) and in our laboratory (6). The red blood cells were suspended at a final hematocrit of $40 \%$ in Krebs-Henseleit buffer which contained (mmol/liter): $118 \mathrm{NaCl}, 4.7 \mathrm{KCl}, 2.0 \mathrm{CaCl}_{2}, 1.2 \mathrm{KH}_{2} \mathrm{PO}_{4}$, $1.2 \mathrm{MgSO}_{4}, 1.2 \mathrm{NaHCO}_{3}, 5.5$ glucose, 1.0 lactate, 0.4 palmitic acid (as a source for free fatty acid) and $4 \mathrm{~g} \%$ free fatty acid-free bovine serum albumin (Catalog No. A-7030, Sigma Chemical Co., St. Louis, MO). The buffer and red cell suspension was prepared fresh daily. Gentamicin $(0.2 \mathrm{mg} / \mathrm{dl})$ was added to the red cell perfusate to retard bacterial growth. The perfusate was equilibrated with $20 \% \mathrm{O}_{2}, 3 \% \mathrm{CO}_{2}, 77 \% \mathrm{~N}_{2}$, to achieve $\mathrm{a}_{2}$ of $100-140 \mathrm{~mm} \mathrm{Hg}$ and $\mathrm{pH}$ of $7.35-7.45$ in all experiments.

After initiation of coronary perfusion, an apical drainage cannula was inserted into the apex of the left ventricle to remove any Thebesian drainage. The cavae were ligated and a cannula was inserted via the ligated pulmonary artery into the right ventricle to collect the coronary venous effluent and completely empty the right ventricle. A pacing electrode connected to a stimulator (Grass Instrument, Co., Quincy, MA) and a thermistor (Yellow Springs Instrument, Co., Yellow Springs, $\mathrm{OH}$ ) were inserted into the right ventricle via the right atrium. Myocardial temperature was maintained at $37^{\circ} \mathrm{C}$ and heart rate was maintained at a physiological rate of $3 \mathrm{~Hz}$ by electrical pacing. A collapsed thin-walled latex balloon was placed in the left ventricle via the left atrium. The balloon was large enough so that no measurable pressure was generated by the balloon itself over the range of LV volume used in this experiment. The heart was then placed in a water-jacketed constant temperature chamber.

The perfusion system consisted of a "venous" reservoir, a variableflow pump, an oxygenator, a water-jacketed "arterial" reservoir, and a filter of $20-\mu \mathrm{m}$ pore size. In this system, the arterial reservoir was pressurized, and coronary perfusion pressure (CPP) was controlled by a valve that adjusted the pressure of the reservoir. Coronary flow was allowed to vary and thus depend on coronary vasomotor autoregulation.

Measurements. Coronary perfusion pressure, LV pressure and its first derivative were recorded continuously on a multichannel physiologic recorder (Gould, Inc., Cleveland, $\mathrm{OH}$ ). $\mathrm{CPP}$ was monitored via a sidearm of the aortic cannula connected to a pressure transducer (Statham model P23Db, Gould, Inc.). LV pressure was measured with short, stiff fluid-filled polyethylene tubing attached to a model P23Db pressure transducer. The frequency response and damping characteristics of this system have been described from this laboratory (22) and satisfy the requirements for accurate measurement of LV pressure and its first derivative. During experiments, LV balloon volume was kept constant so that an increase in LVEDP signified a decrease in LV diastolic chamber distensibility $(4-6,9,10,23)$. LVEDP was measured after transiently turning the pacer off to permit measurement of LVEDP after it reached its nadir during a long diastole.
Coronary blood flow was measured by timed collections of coronary venous effluent collected from the pulmonary artery cannula. Arterial blood gas analysis (Blood Gas Analyzer, Allied Instrumentation Laboratory, Lexington, MA) was performed every $10 \mathrm{~min}$ throughout the protocol. Arterial and coronary venous lactate content was measured by the specific enzymatic method of Apstein et al. (24).

Protocol I: infusion of angiotensin II in the isolated perfused hearts. Rabbits were randomized into two groups: a control group $(n=10)$ which received no drug, and the angiotensin group $(n=10)$ which received angiotensin II at a final concentration of $10^{-8} \mathrm{M}$ (Catalog No. A-9525, Sigma Chemical Co.).

In both groups, $\mathrm{LV}$ balloon volume was initially adjusted to achieve LVEDP of $10 \mathrm{~mm} \mathrm{Hg}$ under control conditions in which CPP was adjusted to a level of $100 \mathrm{~mm} \mathrm{Hg}$. The hearts were paced at $3 \mathrm{~Hz}$ throughout the experiment. The balloon volume was then kept constant for the remainder of the experiment. After 30-min maintenance of hemodynamic steady state, measurements of LV pressure and its first derivative, and coronary flow were made. Samples of the arterial and coronary venous effluent were obtained for determination of lactate content. The samples were collected in 5\% trichloroacetic acid and stored at $4^{\circ} \mathrm{C}$ for subsequent processing. Before the initiation of ischemia, the hearts were then perfused with angiotensin II or no drug for $10 \mathrm{~min}$, and measurements of LV function, coronary flow, and lactate sampling were repeated. From the beginning of angiotensin II infusion to the end of the experiment, coronary venous effluent was not recirculated.

At 10 min after beginning of angiotensin II or no drug perfusion, low-flow ischemia was imposed by reducing CPP to $15 \mathrm{~mm} \mathrm{Hg}$ for 30 min. LV pressure and its first derivative were recorded at the 1 st min and every $5 \mathrm{~min}$ of ischemia. CBF measurement and arterial and venous blood sampling were performed every $5 \mathrm{~min}$. Hearts were then reperfused by restoring CPP to $100 \mathrm{~mm} \mathrm{Hg}$ for $30 \mathrm{~min}$. During the first 5 min of reperfusion, hemodynamic measurements and sampling of arterial and coronary venous effluent were performed every minute and subsequently at $10,15,20$, and $30 \mathrm{~min}$ of reperfusion.

At the end of the experiment, hearts were removed from aortic cannula. After atria and great vessels were removed, total heart and LV weights were measured. The left ventricle was oven-dried for $2 \mathrm{wk}$ for calculation of the LV wet/dry weight ratio.

Protocol II: comparison of hearts with angiotensin II vs. no drug at matched levels of progressive coronary flow reduction. As shown in Results, angiotensin II caused a gradual progressive fall of coronary flow during ischemia compared with the control group with no drug. To distinguish any direct effect of angiotensin II on myocardial function and the effects on coronary flow, another protocol was added. In this protocol ( $n=10$, control hearts with no drug), coronary flow was gradually decreased during low-flow ischemia in order to simulate the change in coronary flow that had occurred in the angiotensin group. To simulate the gradual decrease in coronary flow seen in the angiotensin group, CPP was initially reduced to $15 \mathrm{~mm} \mathrm{Hg}$ and coronary flow was then decreased by a step-wise reduction of coronary perfusion pressure by $1-1.5 \mathrm{mmHg}$ every $5 \mathrm{~min}$ (progressive ischemia group, $n=10$ ). In this progressive ischemia group, the stepwise reduction in CPP was associated with CPP levels of $15 \pm 0.2,13.7 \pm 0.3,12.4 \pm 0.3$, $11.2 \pm 0.6,10.5 \pm 0.6$, and $10.1 \pm 0.8 \mathrm{~mm} \mathrm{Hg}$. After progressive low-flow ischemia, hearts were reperfused for $30 \mathrm{~min}$ as in protocol I.

Protocol III: effects of angiotensin II in the absence of ischemia. To evaluate the effects of angiotensin II alone for the duration of the ischemia-reperfusion protocols described above, four hearts were perfused at a CPP of $100 \mathrm{~mm} \mathrm{Hg}$ with angiotensin II for $70 \mathrm{~min}$, which is equal to the duration of angiotensin exposure in the ischemia and reperfusion protocols cited above.

Analysis of myocardial adenosine triphosphate $(A T P)$ and creatine phosphate $(C P)$ contents. Myocardial ATP and CP contents were determined in three additional groups of hearts $(n=5$ each) at the end of a 30-min period of low-flow ischemia following the protocols described above: a control group which received no drug $(n=5)$, an angiotensin group which received angiotensin II infusion $(n=5)$, and a progressive 
ischemia group which received no drug as well as the gradual reduction of coronary flow during ischemia to simulate the reduction of coronary flow observed in the angiotensin group $(n=5)$.

At the end of the 30-min period of low-flow ischemia during continued perfusion, the heart was quickly trimmed of atria and right ventricular free wall, and the LV was rapidly frozen with Wollenberger aluminum clamps cooled with liquid nitrogen. Each frozen sample was rapidly weighed and pulverized in a mortar in liquid nitrogen. An aliquot of the frozen powder was weighed and then heated $\left(37^{\circ} \mathrm{C}\right)$ in an oven for $48 \mathrm{~h}$ to determine the frozen/dry weight ratio. The remainder of the sample was mixed with $0.6 \mathrm{~N}$ perchloric acid, homogenized, centrifuged, and neutralized with $5 \mathrm{~mol} /$ liter potassium carbonate. The aliquot of neutralized homogenate were placed in preweighed reagent vials and analyzed for ATP by methods of Adams (25). CP was measured by the methods of Altschuld et al. (26) by adding an excess of creatine kinase to the ATP reaction mixture after ATP assay had reached completion. Measurements are expressed as micromoles per gram LV dry weight. In our laboratory, basal values for age-matched New Zealand rabbits are: ATP, $24.1 \pm 0.7 \mu \mathrm{mol} / \mathrm{g} \mathrm{LV}$ dry weight, and $\mathrm{CP}, 49.8 \pm 0.5 \mu \mathrm{mol} / \mathrm{g} \mathrm{LV}$ dry weight.

Statistical analysis. All data are presented as the mean \pm SEM. For each protocol, statistical comparisons between groups at baseline and at preischemia were done using analysis of variance. Comparison between groups in response to 30-min low-flow ischemia and in response to 30-min reperfusion were performed using analysis of variance (ANOVA) for repeated measures. Baseline measurements and 30-min ischemic metabolic measurements (ATP, CP) between two groups were tested by the Student's $t$ test for unpaired data. A $P$ value of $<0.05$ was accepted as the level of significance.

\section{Results}

Effects of angiotensin II in absence of ischemia (Table I). At baseline, there were no differences in LV hemodynamic parameters or coronary flow between the groups. In response to the preischemic 10-min infusion of angiotensin II, there was a mild positive inotropic effect ( $12 \%$ increase in LV developed pressure and $19 \%$ increase in $\mathrm{LV}+\mathrm{d} P / \mathrm{d} t)$ with a slight fall in LVEDP and no difference in $\mathrm{LV}-\mathrm{d} P / \mathrm{d} t$ in the angiotensin II group compared with the control group. The positive inotropic effect was evident at 3 min and reached its maximal effect at 6 min of angiotensin II perfusion with no further changes. Compared with baseline values, there was a slight (8\%) fall in coronary flow in the angiotensin group, but there was no significant difference in coronary flow or aerobic myocardial lactate extraction between the angiotensin II group and the control group before ischemia.

Effects of angiotensin II during low-flow ischemia and reperfusion (Table I). Both groups showed an immediate fall in $\mathrm{LV}$ developed pressure with the onset of low-flow ischemia. LV developed pressure subsequently remained stable in the control group whereas it decreased gradually in the angiotensin II group (Fig. 1, left). At the end of the 30-min period of low-flow ischemia, $\mathrm{LV}$ developed pressure and $\mathrm{LV}+\mathrm{d} P / \mathrm{d} t$ were significantly lower in the angiotensin II group compared with the no-drug control group (Table I). During low-flow ischemia, there was also a marked progressive rise in LVEDP in the angiotensin group compared with the control group (42.0 \pm 5.4 vs. $13.5 \pm 1.5 \mathrm{~mm} \mathrm{Hg}, P<0.01)$ in association with greater depression of $\mathrm{LV}+\mathrm{d} P / \mathrm{d} t$. During the first $5 \mathrm{~min}$ of low-flow ischemia at a constant CPP of $15 \mathrm{~mm} \mathrm{Hg}$, the level of reduced coronary flow was similar in both groups, but coronary flow then progressively decreased in the angiotensin II group. In contrast, the level of coronary flow remained stable during the entire period of ischemia in the control group. Myocardial lactate production as reported by washout during reperfusion was higher in the angiotensin II group (Fig. 2, upper panel).

During the $30 \mathrm{~min}$ of reperfusion imposed by restoring CPP to $100 \mathrm{~mm} \mathrm{Hg}$ (Fig. 1, right), there were no differences in the magnitude of initial reactive hyperemia and subsequent levels of coronary flow at $30 \mathrm{~min}$ of reperfusion. The LV developed pressure recovered to $\sim 73 \%$ of baseline in the control group and to $\sim 62 \%$ of baseline in the angiotensin II group at end of the 30-min reperfusion period. In the control group, there was immediate recovery of the ischemic elevation of LVEDP to baseline, whereas recovery of elevated LVEDP in the angiotensin II group was prolonged.

Comparison of hearts with angiotensin II vs. no drug at matched levels of ischemic coronary flow (Table II). To discriminate the direct effects of angiotensin II on myocardial function from its depressant effects on coronary flow, the angiotensin II group was compared with an additional group of control hearts (progressive ischemia group) in which coronary flow was gradually decreased during the 30 -min period of ischemia to simulate the change in coronary flow observed in the angiotensin II group. In this protocol, coronary flow during ischemia (Fig. 3, left) and myocardial lactate production (Fig. 2) were comparable in both groups. Both groups showed a progressive fall in LV developed pressure (Fig. 3, left) and $\mathrm{LV}+\mathrm{d} P / \mathrm{d} t$ to similar levels at the end of $30 \mathrm{~min}$ of ischemia. In contrast, despite the presence of matched levels of coronary flow throughout the 30-min period of ischemia, the increase in isovolumic LVEDP occurred earlier and was greater in the angiotensin II group compared with the progressive ischemia group (Fig. 3, left). During reperfusion, the recovery of $\mathrm{LV}$ diastolic pressure to baseline was slower and incomplete in the angiotensin II group compared with the progressive ischemia group (Fig. 3, right). At the end of the 30-min period of reperfusion, the recovery of LV developed pressure was less complete and recovery of LV $+\mathrm{d} P / \mathrm{d} t$ was similar in the angiotensin II group compared with the progressive ischemia group. There were no differences in the LV wet/dry weight ratio between the groups.

Effects of angiotensin II without ischemia. To exclude an adverse effect of prolonged angiotensin II perfusion on diastolic function in the absence of ischemia, an additional four hearts were perfused at a CPP of $100 \mathrm{~mm} \mathrm{Hg}$ with $10^{-8} \mathrm{M}$ angiotensin II for $70 \mathrm{~min}$, the total duration of angiotensin II perfusion in the ischemia-reperfusion protocols cited above. Angiotensin II perfusion was associated with a mild inotropic effect similar to that described above. During $70 \mathrm{~min}$ of angiotensin II perfusion in the absence of ischemia, there was no significant change in LVEDP $(9.8 \pm 0.3$ to $7.5 \pm 0.6 \mathrm{~mm} \mathrm{Hg}, \mathrm{NS})$ or $\mathrm{LV}-\mathrm{d} P / \mathrm{d} t$ (from $1,145 \pm 69$ to $1,143 \pm 142 \mathrm{~mm} \mathrm{Hg} / \mathrm{s}, \mathrm{NS}$ ).

Myocardial ATP and CP levels at end-ischemia. LV myocardial ATP and CP content were measured at the end of 30 min of low-flow ischemia at CPP of $15 \mathrm{~mm} \mathrm{Hg}$ without reperfusion in five additional hearts in each of the three groups: the control group with no drug, the angiotensin II group, and the progressive ischemia group (hearts with no drug in which coronary flow was progressively reduced during the ischemic period to match levels observed in the angiotensin II group).

Within each group, the measurements of $\mathrm{LV}$ function and coronary flow at baseline and in response to low-flow ischemia were similar to those observed in hearts exposed to both ischemia and reperfusion. At the end of $30 \mathrm{~min}$ of low-flow isch- 

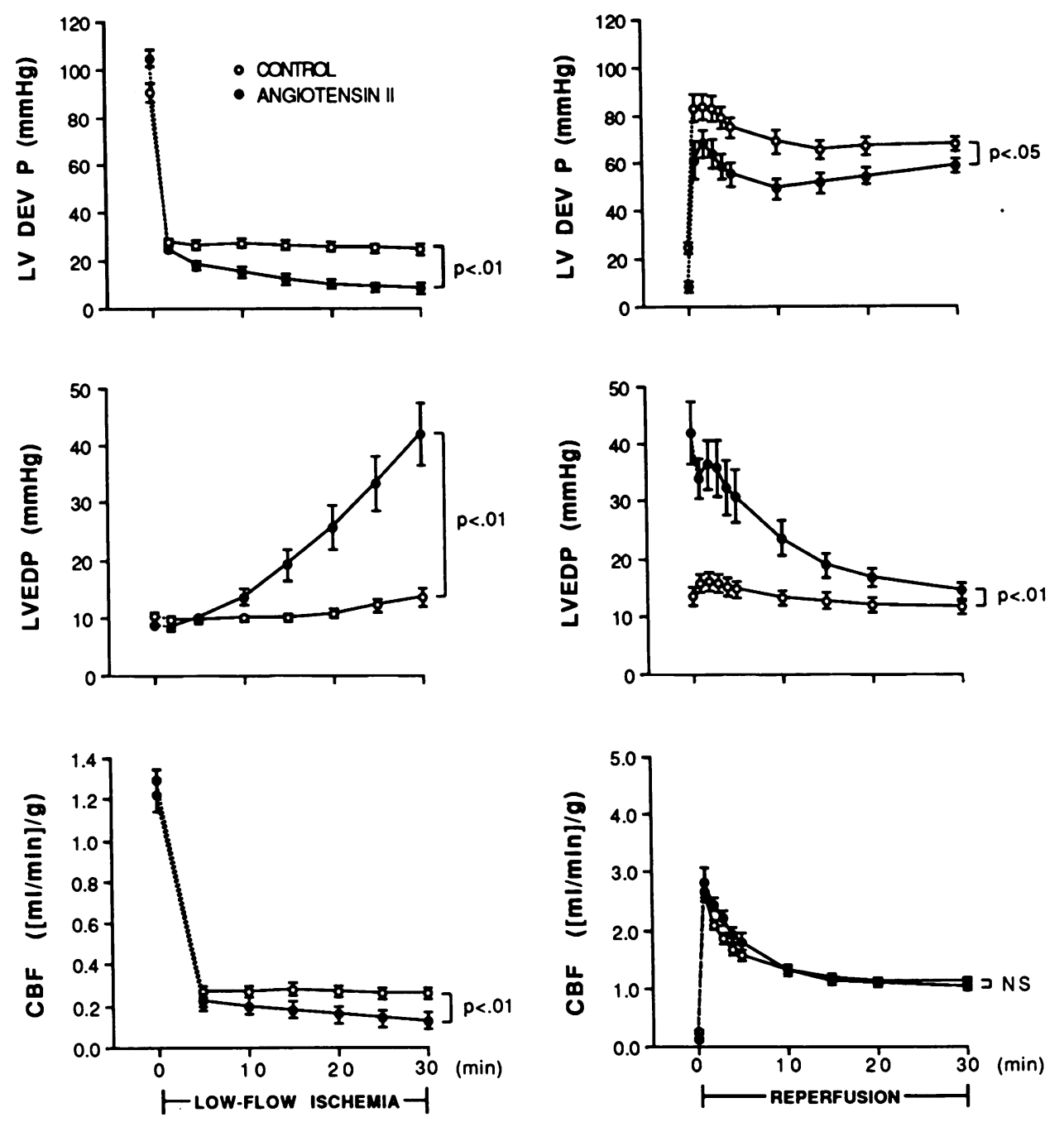

Figure 1. The left panel shows hemodynamic parameters immediately before ischemia and during 30 min of low-flow ischemia in the angiotensin group (hearts perfused with angiotensin II for $10 \mathrm{~min}$ before ischemia, during ischemia, and during reperfusion) and the control group (hearts perfused with no drug). The right panel shows hemodynamic parameters during $30 \mathrm{~min}$ of reperfusion.

emia, the LVEDP rose to $12.8 \pm 1.1 \mathrm{~mm} \mathrm{Hg}$ in the control group, $35.8 \pm 3.1 \mathrm{~mm} \mathrm{Hg}$ in the angiotensin II group, and $19.6 \pm 3.2 \mathrm{~mm} \mathrm{Hg}$ in the progressive ischemia group. The biochemical measurements are summarized in Table III. Compared with basal values for normal age-matched rabbits in our laboratory, low-flow ischemia resulted in a $60 \%$ reduction in ATP content and a $75 \%$ reduction in CP content. There were no differences between the groups. The LV frozen/dry weight ratio measurements were also similar between the groups.

\section{Discussion}

Angina pectoris in patients with coronary artery disease is associated with an acute and reversible depression of myocardial relaxation and diastolic distensibility as well as systolic contractility (1-3). In experiments that attempt to model diastolic function during transient ischemia, it is critical to differentiate the effects of primary no-flow ischemia and low-flow demand ischemia $(4,6,22,27)$. Early in the course of no-flow ischemia induced by total cessation of coronary flow, there is an immediate and complete loss of contractile function whereas diastolic dysfunction is minimal because of the loss of coronary turgor and the depressant effects of proton and inorganic phosphate accumulation on calcium-dependent force development. In contrast, during ischemia which occurs in the presence of con- tinued but severely restricted coronary flow, a reduced level of contractile function is preserved whereas impaired diastolic relaxation and the elevation of diastolic pressure occur rapidly. We have previously shown that these acute and reversible changes in diastolic function can be simulated in the isolated red cell-perfused heart model used in this experiment in which the critical feature is that global ischemia is produced at a reduced level of coronary blood flow sufficient to sustain contractile function (4-6).

The acute and reversible changes in diastolic function characteristic of low-flow ischemia are postulated to be related to modest levels of high-energy phosphate depletion in association with the impaired restoration of normal diastolic intracellular calcium levels $(7,8,27)$. Consistent with this view, the changes in diastolic relaxation and distensibility that occur during low-flow ischemia are modified by interventions which alter calcium availability or energy demand and availability $(4-6,28)$ and amplified in hypertrophied hearts with intrinsic impairment of calcium homeostasis $(10,11)$.

However, the potential contribution of neurohumoral factors, such as the renin-angiotensin system, on the contractile and metabolic response of the heart to low-flow ischemia has received little attention. This is of interest since there is evidence that angiotensin II has the potential to modify cardiac physiology via the systemic circulation as well as newly identi- 
Table I. Protocol I: LV Hemodynamic Parameters

\begin{tabular}{|c|c|c|c|c|}
\hline & Baseline & $\begin{array}{l}\text { Preischemia } \\
\text { (10 min) }\end{array}$ & $\begin{array}{l}\text { Ischemia } \\
(30 \mathrm{~min})\end{array}$ & $\begin{array}{c}\text { Reperfusion } \\
\text { (30 min) }\end{array}$ \\
\hline \multicolumn{5}{|c|}{ LVEDP $(m m \mathbf{H g})$} \\
\hline C group & $10.8 \pm 0.3$ & $10.4 \pm 0.4$ & $13.5 \pm 1.5$ & $11.4 \pm 1.1$ \\
\hline AT group & $10.8 \pm 0.2$ & $8.8 \pm 0.3$ & $42.0 \pm 5.4$ & $14.3 \pm 1.5$ \\
\hline$P$ & NS & $<0.05$ & $<0.01$ & $<0.01$ \\
\hline \multicolumn{5}{|c|}{ LVSP $(m m ~ H g)$} \\
\hline $\mathrm{C}$ group & $102.7 \pm 3.2$ & $101.5 \pm 3.6$ & $38.3 \pm 1.4$ & $79.0 \pm 2.7$ \\
\hline AT group & $104.4 \pm 3.4$ & $113.6 \pm 3.4$ & $50.3 \pm 4.4$ & $72.9 \pm 1.7$ \\
\hline$P$ & NS & $<0.05$ & $<0.01$ & NS \\
\hline \multicolumn{5}{|c|}{ LV DEV P $(\mathrm{mm} \mathrm{Hg})$} \\
\hline$C$ group & $91.9 \pm 3.3$ & $91.1 \pm 3.9$ & $24.8 \pm 2.2$ & $67.6 \pm 3.3$ \\
\hline AT group & $93.6 \pm 3.5$ & $104.8 \pm 3.4$ & $8.3 \pm 2.1$ & $58.6 \pm 2.9$ \\
\hline$P$ & NS & $P<0.05$ & $P<0.01$ & $P<0.05$ \\
\hline \multicolumn{5}{|c|}{$\begin{array}{l}\mathrm{LV}+\mathrm{d} P / \mathrm{d} t \\
\quad(m m H g / s)\end{array}$} \\
\hline C group & $1,465 \pm 43$ & $1,452 \pm 59$ & $477 \pm 31$ & $1,081 \pm 58$ \\
\hline AT group & $1,625 \pm 63$ & $1,902 \pm 73$ & $159 \pm 44$ & $1,093 \pm 54$ \\
\hline$P$ & NS & $<.001$ & $<.01$ & NS \\
\hline \multicolumn{5}{|c|}{$\begin{array}{l}\mathrm{LV}-\mathrm{d} P / \mathrm{d} t \\
\quad(-m m H g / s)\end{array}$} \\
\hline $\mathrm{C}$ group & $1,123 \pm 39$ & $1,136 \pm 46$ & $379 \pm 37$ & $924 \pm 45$ \\
\hline AT group & $1,087 \pm 27$ & $1,235 \pm 41$ & $121 \pm 26$ & $797 \pm 43$ \\
\hline$P$ & NS & NS & $<0.01$ & $P<0.01$ \\
\hline \multicolumn{5}{|l|}{$\mathrm{CF}(\mathrm{ml} / \mathrm{min})$} \\
\hline C group & $3.92 \pm 0.30$ & $3.92 \pm 0.30$ & $0.78 \pm 0.06$ & $3.35 \pm 0.25$ \\
\hline AT group & $4.16 \pm 0.37$ & $3.83 \pm 0.33$ & $0.38 \pm 0.05$ & $3.34 \pm 0.42$ \\
\hline$P$ & NS & NS & $<0.01$ & NS \\
\hline \multicolumn{5}{|c|}{$\mathrm{CF}(\mathrm{ml} / \mathrm{min} / \mathrm{gLV})$} \\
\hline $\mathrm{C}$ group & $1.29 \pm 0.06$ & $1.29 \pm 0.06$ & $0.26 \pm 0.02$ & $1.12 \pm 0.07$ \\
\hline AT group & $1.32 \pm 0.08$ & $1.22 \pm 0.08$ & $0.13 \pm 0.02$ & $1.04 \pm 0.08$ \\
\hline$P$ & NS & NS & $<0.01$ & NS \\
\hline
\end{tabular}

Abbreviations: C group, control hearts without drug; AT group, hearts perfused with angiotensin II during the periods of 10-min preischemia, 30-min ischemia, and 30-min reperfusion; LVEDP, left ventricular end-diastolic pressure; LVSP, left ventricular systolic pressure; LV DEV P, LV developed pressure; LV $+\mathrm{d} P / \mathrm{d} t$, peak positive left ventricular $\mathrm{d} P / \mathrm{d} t ; \mathrm{LV}-\mathrm{d} P / \mathrm{d} t$, peak negative left ventricular $\mathrm{d} P / \mathrm{d} t ; \mathrm{CF}$, coronary flow, absolute values and flow per gram left ventricular wet weight. Values at single time points at baseline and preischemia were compared by analysis of variance. Comparison between the groups in response to 30-min low-flow ischemia and in response to 30-min reperfusion was done using analysis of variance (ANOVA) for repeated measures.

fied local cardiac angiotensin II activation (12-16). High-affinity angiotensin II receptors have been identified in the conduction system, endothelium, and myocytes in several species, including the rabbit $(12,14,15,29)$. The cardiac effects of angiotensin II appear to include effects on myocardial contractility, coronary vasomotor tone, and the modulation of cardiac hypertrophy which may facilitate adaptation of the well-oxygenated heart to stress $(12,15,16,30,31)$.

In contrast, there is indirect evidence that suggests deleterious effects of angiotensin II on the heart subjected to ischemia and reperfusion. Recent studies have shown the beneficial effect of the specific inhibition of ACE and angiotensin II activation against ischemic and reperfusion injury $(18,19,20,32)$. To study the direct effects of angiotensin II on myocardial contractile function and metabolism during ischemia and reperfusion, we compared the effects of $30 \mathrm{~min}$ of low-flow ischemia and reperfusion in beating, isovolumic red cell-perfused rabbit hearts in the presence and absence of $10^{-8} \mathrm{M}$ angiotensin II. Our new observations indicate that angiotensin II directly impairs LV diastolic function during low-flow ischemia and its recovery during reperfusion, independent of the depressant ef-
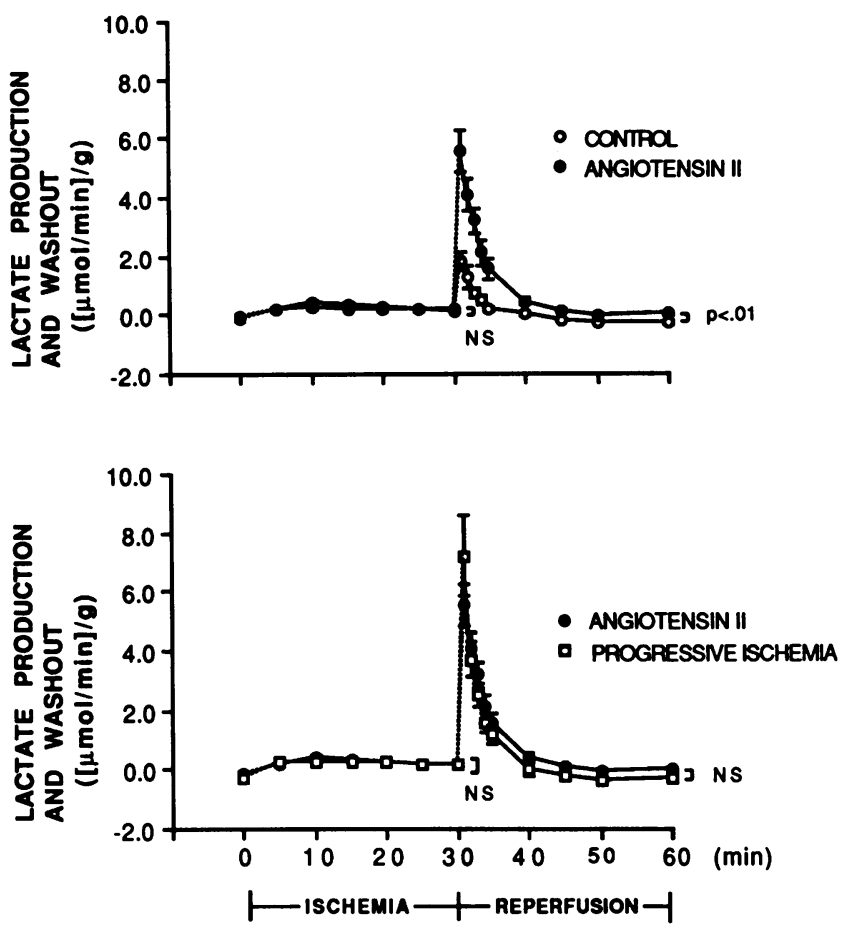

Figure 2. Myocardial lactate production as reported by myocardial lactate washout during reperfusion. Upper panel: control group (hearts perfused with no drug) and angiotensin group (hearts perfused with angiotensin II through ischemia and reperfusion). Lower panel: angiotensin group and progressive ischemia group (hearts perfused with no drug in which coronary flow was progressively reduced during ischemia to match the levels in the angiotensin-treated hearts).

fects of angiotensin II on coronary vasomotor tone during ischemia and the magnitude of high-energy phosphate depletion.

Effects of angiotensin II in nonischemic isolated hearts. Under well-oxygenated conditions, we perfused isolated paced red cell-perfused rabbit hearts with a concentration of angiotensin II which produced a mild positive inotropic effect, no adverse effect on diastolic function, and minimal coronary vasoconstriction in the presence of a normal aerobic pattern of lactate metabolism. Angiotensin II's constricting effect on vasomotor tone has been observed in all species studied to date, whereas the positive inotropic effect of angiotensin II that we observed is consistent with previous studies in the rabbit, chick, dog, and cat (12, 33-36) and humans (17). It is noteworthy and not well understood that angiotensin II causes a negligible or depressant inotropic effect in some species such as the rat and guinea pig $(15,16,30,37)$. This contrasts with the positive inotropic and lusitropic effects of $\beta$-adrenergic agonists observed across species and suggests that the physiologic effects of angiotensin II are not predominantly mediated by cyclic AMPdependent pathways (30).

Effects of angiotensin II during low-flow ischemia and reperfusion. The imposition of ischemia imposed by reducing coronary perfusion pressure from 100 to $15 \mathrm{~mm} \mathrm{Hg}$ was immediately followed by a reduction in coronary flow to a level of $\sim 20 \%$ of baseline and a reduction in parameters of contractile function to $\sim 25 \%$ of baseline at $1 \mathrm{~min}$ of ischemia. Consistent with prior studies using this model of transient low-flow ischemia $(4,5,11)$, coronary flow and all parameters of systolic function remained stable during the remainder of the 30-min 
Table II. Protocol II: LV Hemodynamic Parameters

\begin{tabular}{|c|c|c|c|c|}
\hline & Baseline & $\begin{array}{l}\text { Preischemia } \\
\text { (10 min) }\end{array}$ & $\begin{array}{l}\text { Ischemia } \\
\text { (30 min) }\end{array}$ & $\begin{array}{l}\text { Reperfusion } \\
\text { (30 min) }\end{array}$ \\
\hline \multicolumn{5}{|c|}{ LVEDP $(m m ~ H g)$} \\
\hline PI group & $10.0 \pm 0.1$ & $9.6 \pm 0.3$ & $19.9 \pm 1.3$ & $10.9 \pm 0.5$ \\
\hline AT group & $10.8 \pm 0.2$ & $8.8 \pm 0.3$ & $42.0 \pm 5.4$ & $14.3 \pm 1.5$ \\
\hline$P$ & NS & NS & $<0.01$ & $<0.01$ \\
\hline \multicolumn{5}{|c|}{$\operatorname{LVSP}(\mathrm{mm} \mathrm{Hg})$} \\
\hline PI group & $110.6 \pm 4.3$ & $107.9 \pm 4.4$ & $30.8 \pm 1.8$ & $81.0 \pm 3.9$ \\
\hline AT group & $104.4 \pm 3.4$ & $113.6 \pm 3.4$ & $50.3 \pm 4.4$ & $72.9 \pm 1.7$ \\
\hline$P$ & NS & NS & $<0.01$ & NS \\
\hline \multicolumn{5}{|c|}{ LV DEV P $(\mathrm{mm} \mathrm{Hg})$} \\
\hline PI group & $100.6 \pm 4.4$ & $98.3 \pm 4.4$ & $10.9 \pm 1.2$ & $70.1 \pm 3.8$ \\
\hline AT group & $93.6 \pm 3.5$ & $104.8 \pm 3.4$ & $8.3 \pm 2.1$ & $58.6 \pm 2.9$ \\
\hline$P$ & NS & NS & NS & $<0.01$ \\
\hline \multicolumn{5}{|c|}{$\begin{array}{l}\mathrm{LV}+\mathrm{d} P / \mathrm{d} t \\
\quad(m m H g / s)\end{array}$} \\
\hline PI group & $1,717 \pm 105$ & $1,659 \pm 107$ & $242 \pm 26$ & $1,156 \pm 75$ \\
\hline AT group & $1,625 \pm 63$ & $1,902 \pm 73$ & $159 \pm 44$ & $1,093 \pm 54$ \\
\hline$P$ & NS & NS & NS & NS \\
\hline \multicolumn{5}{|c|}{$\begin{array}{l}\mathrm{LV}-\mathrm{d} P / \mathrm{d} t \\
\quad(-m m H g / s)\end{array}$} \\
\hline PI group & $1,160 \pm 58$ & $1,163 \pm 56$ & $155 \pm 21$ & $947 \pm 55$ \\
\hline AT group & $1,087 \pm 27$ & $1,235 \pm 41$ & $121 \pm 26$ & $797 \pm 43$ \\
\hline$P$ & NS & NS & NS & $<0.05$ \\
\hline \multicolumn{5}{|l|}{$\mathrm{CF}(\mathrm{ml} / \mathrm{min})$} \\
\hline PI group & $3.91 \pm 0.28$ & $3.85 \pm 0.30$ & $0.35 \pm 0.01$ & $3.45 \pm 0.39$ \\
\hline AT group & $4.16 \pm 0.37$ & $3.83 \pm 0.33$ & $0.38 \pm 0.05$ & $3.34 \pm 0.42$ \\
\hline$P$ & NS & NS & NS & NS \\
\hline \multicolumn{5}{|c|}{$\mathrm{CF}(\mathrm{ml} / \mathrm{min} / \mathrm{g} L V)$} \\
\hline PI group & $1.36 \pm 0.07$ & $1.34 \pm 0.08$ & $0.12 \pm 0.01$ & $1.19 \pm 0.11$ \\
\hline AT group & $1.32 \pm 0.08$ & $1.22 \pm 0.08$ & $0.13 \pm 0.02$ & $1.04 \pm 0.08$ \\
\hline$P$ & NS & NS & NS & $<0.05$ \\
\hline
\end{tabular}

Abbreviations: PI group, progressive ischemia group consisting of control hearts with no drug in which coronary flow was progressively reduced during the 30-min period of ischemia to match levels in the AT group; AT group, hearts perfused with angiotensin II which was begun 10 min preischemia and continued throughout ischemia and reperfusion. Other terms, same as Table 1.

period of ischemia in the control hearts with no drug. In contrast, the hearts perfused with angiotensin II showed a gradual progressive fall in coronary flow during ischemia in association with greater myocardial lactate production and washout. During reperfusion, the magnitude of reactive hyperemia and level of coronary flow at 30 min of recovery were similar in both groups. These observations suggest that a level of angiotensin II that caused minimal changes in coronary vasomotor tone under oxygenated conditions promoted inappropriate coronary vasoconstriction and exacerbation of the magnitude of ischemia in the setting of a reduced coronary perfusion pressure. In the hearts perfused with angiotensin II compared with control hearts, a positive inotropic effect was no longer evident at $1 \mathrm{~min}$ of ischemia and this group showed a progressive depression of contractile function during low-flow ischemia. During reperfusion, recovery of systolic function was slower and incomplete in the angiotensin group compared with the control groups. These results contrast with prior experiments from our laboratory which showed that isoproterenol infusion at a dose that caused a comparable mild inotropic effect under oxygenated conditions was associated with a favorable effect on both systolic and diastolic function during low-flow ischemia (5).

During 30 min of low-flow ischemia, angiotensin II caused a more rapid and severe increase in isovolumic LVEDP of $\sim 30 \mathrm{~mm} \mathrm{Hg}$ in association with a greater depression of $\mathrm{LV}$ $-\mathrm{d} P / \mathrm{d} t$, and very delayed recovery during reperfusion in comparison with control hearts with no drug. This deleterious effect of angiotensin II on diastolic function in the angiotensin II group is unlikely to be explained by exaggerated energy demand in that heart rate was constant and parameters of contractile function were lower during ischemia in the angiotensin group relative to the control group, nor can it be explained by the extrinsic factors of coronary turgor or edema since coronary flow was lower and the wet/dry ratio was similar in the angiotensin group compared with control hearts.

Comparison of the effects of angiotensin II vs. no drug at matched levels of ischemic coronary flow. To distinguish any direct myocardial effect of angiotensin II on LV contractile and lusitropic function from its depressant effects on coronary flow during low-flow ischemia, an additional group of control hearts with no drug was studied in which coronary flow was progressively reduced during the 30 -min period of ischemia. With this experimental strategy, coronary flow throughout the 30 -min period of ischemia was similar in both groups. Although differences in subendocardial flow cannot be excluded, myocardial lactate production and washout were virtually identical in the angiotensin II group and this control group with progressive ischemia. At matched levels of progressive reduction of coronary flow, there was now a similar magnitude of depression of LV developed pressure in response to $30 \mathrm{~min}$ of ischemia. However, during ischemia, angiotensin II compared with no drug caused a more rapid and severe elevation of isovolumic LVEDP whose recovery was slower and incomplete during the 30-min period of reperfusion.

Analysis of left ventricular myocardial high energy phosphate levels in additional subsets of hearts at the end of $30 \mathrm{~min}$ of ischemia showed that the adverse effect of angiotensin II on ischemic diastolic function cannot be explained by a greater depletion of myocardial high-energy phosphate compounds since the levels of ATP and CP were similar between hearts treated with angiotensin compared with no drug. There is evidence that ATP produced by anaerobic glycolysis, which cannot be distinguished by measurements of total myocardial ATP and $\mathrm{CP}$ content, may exert a protective effect against diastolic dysfunction during ischemia and hypoxia $(6,38)$. This metabolic pathway cannot explain the adverse effect of angiotensin II on diastolic function because glycolytic ATP generation as reported by myocardial lactate production was not depressed relative to control hearts.

Significance of angiotensin and ischemic dysfunction. These observations of the deleterious effect of angiotensin II on ischemic diastolic function are consistent with other studies which have examined the potential protection of ACE inhibitors during experimental ischemia (18-20). In comparison with our red-cell perfused model of low-flow ischemia, $\mathrm{Li}$ and Chen (20) studied the effects of enalapril administration in rats $1 \mathrm{~h}$ before sacrifice of the animal and the subsequent imposition of global no-flow ischemia and reperfusion in an isolated buffer-perfused heart model. They observed that hearts from rats which were treated with ACE inhibition demonstrated improved reactive coronary flow associated with improved contractile force during reperfusion. This is consistent with our observation (protocol I) in red cell-perfused hearts subjected to low-flow ischemia that angiotensin II directly promoted vasoconstriction during low-flow ischemia in association with greater depression of systolic and diastolic function during 

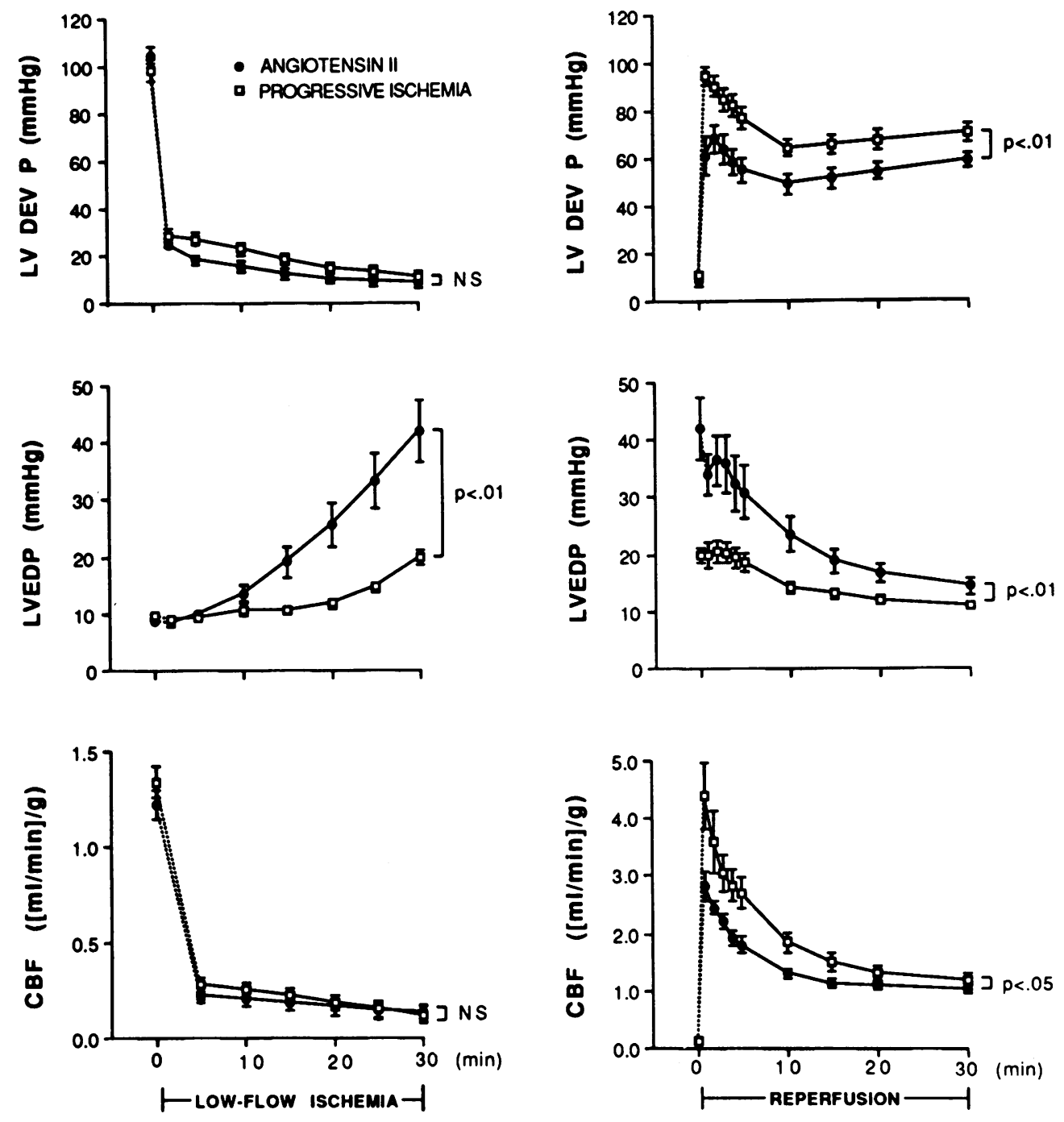

Figure 3. The left panel shows hemodynamic parameters immediately before ischemia and during $30 \mathrm{~min}$ of low-flow ischemia in the angiotensin group and the progressive ischemia group (control hearts with no drug in which coronary flow was progressively reduced during ischemia to match levels in the angiotensin-treated hearts). The right panel shows hemodynamic parameters during $30 \mathrm{~min}$ of reperfusion.

both ischemia and reperfusion compared with hearts treated with no drug. Additionally, we have demonstrated a beneficial effect of ACE inhibition with enalaprilat on ischemic diastolic dysfunction during low-flow ischemia in rat hearts with pressure-overload hypertrophy (32), in which local cardiac angiotensin II activation is increased (16). Furthermore, recent studies in patients with coronary artery disease suggest that $\mathrm{ACE}$

Table III. Tissue ATP and CP Contents at the End of 30-min Low-Flow Ischemia

\begin{tabular}{lcc}
\hline \multicolumn{1}{c}{ Group } & ATP & CP \\
\hline & \multicolumn{2}{c}{$\mu$ mol/g $L V$ dry weight } \\
C group $(n=5)$ & $10.6 \pm 0.7$ & $13.6 \pm 2.3$ \\
AT group $(n=5)$ & $9.1 \pm 1.3$ & $13.3 \pm 3.4$ \\
PI group $(n=5)$ & $9.8 \pm 0.6$ & $13.3 \pm 1.2$ \\
\hline
\end{tabular}

Abbreviations: C, control group; AT, angiotensin group; PI, progressive ischemia group. (Normative values in this laboratory in agematched rabbits are: ATP, $24.1 \pm 0.7 \mu \mathrm{mol} / \mathrm{g} \mathrm{LV}$ dry weight; CP, $49.8 \pm 0.5 \mu \mathrm{mol} / \mathrm{g} \mathrm{LV}$ dry weight.) There were no significant differences between the groups for levels of ATP or CP. inhibition causes improvement in left ventricular diastolic function during ischemia (39), and that this effect is especially prominent in patients with ischemic heart disease during the stress of exercise (40). Further clinical studies employing intracoronary administration of ACE inhibitors will be needed to demonstrate a protective effect of ACE inhibitors on ischemic diastolic dysfunction and to distinguish effects of systemic angiotensin II from local cardiac activation.

Potential mechanisms. There are several potential mechanisms which could be responsible for the adverse effect of angiotensin II on diastolic function during low-flow ischemia and subsequent reperfusion. In this isolated heart model, it is unlikely that the effects of angiotensin II were mediated by facilitation of sympathetic neurotransmitter release (41). However, a limitation of this study is that this indirect effect of angiotensin II may be more dominant in vivo.

The subcellular mechanisms of angiotensin II signal transduction are incompletely understood. There is recent evidence that the effects of angiotensin II on cardiac performance and growth may be dependent on receptor-mediated activation of phosphoinositide second messengers and changes in the mobilization and reuptake of cytosolic $\mathrm{Ca}^{2+}(31,37,42-44)$ as well as myofibrillar $\mathrm{Ca}^{2+}$ sensitivity (45). In the setting of low-flow ischemia and hypoxia, in which there is a prompt increase in 
intracellular free $\mathrm{Ca}^{2+}(7,8)$, the activation of inositol triphosphate and protein kinase $\mathrm{C}$ may exacerbate dysfunctional $\mathrm{Ca}^{2+}$ homeostasis, and further impair myocardial relaxation. In addition, there is evidence that angiotensin II may promote intracellular alkalinization by enhanced $\mathrm{Na}^{+}-\mathrm{H}^{+}$exchange secondary to protein kinase $C$ activation $(31,46-48)$. Preliminary studies from our laboratories using isolated rabbit hearts and rabbit myocytes loaded with the $\mathrm{Ca}^{2+}$ indicator Indo-I AM support the notion that the effects of angiotensin II on contractility may be predominantly mediated by a alkalosis-induced increase in myofilament $\mathrm{Ca}^{2+}$ sensitivity rather than a predominant increase in free activator $\mathrm{Ca}^{2+}(49)$. During energy depletion, intracellular acidosis protects against the development of calcium-activated force and diastolic dysfunction $(28,50,51)$. Thus, future studies will need to be done to test the hypothesis that the adverse effect of angiotensin II on diastolic function during ischemia is mediated by intracellular alkalosis and an increased myofilament $\mathrm{Ca}^{2+}$ sensitivity. The greater left ventricular systolic pressure in the angiotensin II group at baseline is consistent with an increase in cytosolic $\mathrm{Ca}^{2+}$ or a change in sensitivity to $\mathrm{Ca}^{2+}$.

Clinical implications. These experiments raise the possibility that angiotensin II may play a contributory role in the development of congestive heart failure secondary to acute diastolic dysfunction during transient ischemia in patients. In such patients, an increase in angiotensin II via the systemic renin-angiotensin system or via local ACE-specific or nonspecific cardiac angiotensin II-forming pathways (51) could promote adverse changes in both coronary vasomotor tone and diastolic relaxation. The adverse effects of angiotensin II during ischemia may be particularly prominent in the setting of cardiac hypertrophy, in which the cardiac activation of angiotensin II appears to be enhanced (16). Such hypertrophy and local activation of angiotensin II may occur in a regional distribution in postinfarction remodeling associated with coronary artery disease (52) in addition to the global hypertrophy that occurs in response to pressure overload. Thus, many patients may be predisposed to develop ischemic diastolic dysfunction, mediated at least in part by the actions of the renin-angiotensin system on the heart.

\section{Acknowledgments}

We appreciate the technical assistance of Mr. Soeun Ngoy in the preparation of the red cell perfusate, and the assistance of Ms. Barbara Zillman in the preparation of the manuscript.

This study was supported in part by grants from the National Heart, Lung, and Blood Institute (HL-28939, to Dr. Lorell and HL-31807 and HL-38189 to Dr. Apstein), and an Established Investigatorship and Grant-in-Aid of the American Heart Association (Dr. Lorell).

\section{References}

1. Mann, T. S., S. Goldberg, and W. Grossman. 1979. Factors contributing to altered left ventricular diastolic properties during angina pectoris. Circulation. 59:14-20.

2. Carroll, J. D., O. M. Hess, H. O. Hirzel, and H. P. Krayenbuehl. 1983 Exercise-induced ischemia: the influence of altered relaxation on early diastolic pressures. Circulation. 67:521-528.

3. Bourdillon, P. D., B. H. Lorell, I. Mirsky, W. J. Paulus, J. Wynne, and W. Grossman. 1983. Increased regional myocardial stiffness of the left ventricle during pacing-induced angina in man. Circulation. 67:316-323.

4. Isoyama, S., C. S. Apstein, L. Wexler, W. N. Grice, and B. H. Lorell. 1987. Acute decrease in left ventricular diastolic chamber distensibility during simulated angina in isolated hearts. Circ. Res. 61:925-933.
5. Lorell, B. H., S. Isoyama, W. N. Grice, E. O. Weinberg, and C. S. Apstein. 1988. Effects of ouabain and isoproterenol on left ventricular diastolic function during low-flow ischemia in isolated, blood-perfused rabbit hearts. Circ. Res. 63:457-467.

6. Eberli, F. R., E. O. Weinberg, W. N. Grice, G. L. Horowitz, and C. S. Apstein. 1991. Protective effect of increased glycolytic substrate against systolic and diastolic dysfunction and increased coronary resistance from prolonged global underperfusion and reperfusion in isolated rabbit hearts perfused with erythrocyte suspensions. Circ. Res. 68:466-481.

7. Lee, H. C., R. Mohabir, N. Smith, M. R. Franz, and W. T. Clusin. 1988. Effect of ischemia on calcium-dependent fluorescence transients in rabbit hearts containing Indo-1: correlations with monophasic action potentials and contraction. Circulation. 78:1047-1059.

8. Kihara, Y., W. Grossman, and J. P. Morgan. 1989. Direct measurement of changes in $\left[\mathrm{Ca}^{2+}\right]_{\mathrm{i}}$ during hypoxia, ischemia, and reperfusion of the intact mammalian heart. Circ. Res. 65:1029-1044.

9. Lorell, B. H., L. F. Wexler, S. Momomura, E. O. Weinberg, and C. S. Apstein. 1986. The influence of pressure-overload left ventricular hypertrophy on diastolic properties during hypoxia in isovolumically contracting rat hearts. Circ. Res. 58:653-663.

10. Lorell, B. H., W. N. Grice, and C. S. Apstein. 1989. Influence of hypertension with minimal hypertrophy during demand ischemia. Hypertension. 13:362370 .

11. Lorell, B. H., W. N. Grice, and C. S. Apstein. 1989. Impaired diastolic tolerance to low-flow ischemia in blood-perfused hypertrophied rat hearts. $\mathrm{Circu}$ lation. 80(Suppl. II):97.

12. Dzau, V. J. 1988. Cardiac renin-angiotensin system: molecular and functional aspects. Am. J. Med. 84:22-27.

13. Kanapuli, S. P., and A. Kumar. 1987. Molecular cloning of human angiotensin cDNA and evidence for the presence of its mRNA in rat heart. Circ. Res. 60:786-790.

14. Baker, K. M., C. P. Campanile, G. J. Trachte, and M. J. Peach. 1984. Identification and characterization of the rabbit angiotensin II myocardial receptor. Circ. Res. 54:286-293.

15. Lindpaintner, K., M. Jin, M. J. Wilhelm, F. Susuki, W. Linz, B. A. Schoelkens, and D. Ganten. 1988. Intracardiac generation of angiotensin and its physiologic role. Circulation. 77(Suppl. I):1-18.

16. Schunkert, H., V. J. Dzau, S. S. Tang, A. T. Hirsch, C. S. Apstein, and B. H. Lorell. 1990. Increased rat cardiac angiotensin converting enzyme activity and mRNA expression in pressure overload left ventricular hypertrophy: effects on coronary resistance, contractility, and relaxation. J. Clin. Invest. 86:19131920.

17. Foult, J. M., O. Tavolaro, I. Antony, and A. Nitenberg. 1988. Direct myocardial and coronary effects of enalaprilat in patients with dilated cardiomyopathy: assessment by a bilateral intracoronary infusion technique. Circulation. 77:337-344.

18. Van Gilst, W. H., P. A. De Graeff, H. Wesseling, and C. D. J. De Langen. 1986. Reduction of reperfusion arrhythmias in the ischemic isolated rat heart by angiotensin-converting enzyme inhibitors: a comparison of captopril, enalapril and HOE 498. J. Cardiovasc. Pharmacol. 8:722-728.

19. Linz, W., B. A. Schölkens, and Y.-F. Han. 1986. Beneficial effects of the converting enzyme inhibitor, ramipril, in ischemic rat hearts. $J$. Cardiovasc. Pharmacol. 8(Suppl. 10):S91-99.

20. Li, K., and X. Chen. 1987. Protective effects of captopril and enalapril on myocardial ischemia and reperfusion damage of rat. J. Mol. Cell. Cardiol. 19:909-915.

21. Marshall, R. C., and D. Y. Zhang. 1988. Correlation of contractile dysfunction with oxidative energy production and tissue high energy phosphate stores during partial coronary flow disruption in rabbit heart. J. Clin. Invest. 82:86-95.

22. Serizawa, T., W. M. Vogel, C. S. Apstein, and W. Grossman. 1981. Comparison of acute alterations in left ventricular diastolic chamber stiffness induced by hypoxia and ischemia. J. Clin. Invest. 68:91-102.

23. Apstein, C. S., M. Mueller, and W. B. Hood, Jr. 1977. Ventricular contracture and compliance changes with global ischemia and reperfusion, and their effect on coronary resistance in the rat. Circ. Res. 41:206-217.

24. Apstein, C. S., E. Puchner, and N. Brachfeld. 1970. Improved automated lactate determination. Anal. Biochem. 38:20-34.

25. Adams, H. 1963. Adenosine 5'-triphosphate determination with phosphoglycerate kinase. In Methods of Enzymatic Analysis. H. V. Bergmeyer, editor. Academic Press, Inc. New York. 539-543.

26. Altschuld, R. A., A. Weiss, F. A. Kruger, and A. M. Weissler. 1969 Anaerobic performance and metabolism of hyperthyroid heart. J. Clin. Invest 48:1905-1913.

27. Apstein, C. S., and W. Grossman. 1987. Opposite initial effects of supply and demand ischemia on left ventricular diastolic compliance: the ischemia-diastolic paradox. J. Mol. Cell. Cardiol. 19:119-128.

28. Momomura, S., J. S. Ingwall, J. A. Parker, P. Sahagian, J. J. Ferguson, and W. Grossman. 1985. The relationships of high energy phosphates, tissue $\mathrm{pH}$ and 
regional blood flow to diastolic distensibility in the ischemic dog myocardium. Circ. Res. 57:822-835.

29. Wright, G. B., R. W. Alexander, L. S. Ekstein, and M. A. Gimbrone. 1983. Characterization of the rabbit ventricular myocardial receptor for angiotensin II. Mol. Pharmacol. 24:213-221.

30. Allen, I. S., N. M. Cohen, T. S. Dhallen, S. T. Gaa, W. J. Lederer, and T. B. Rogers. 1988. Angiotensin II increases spontaneous contractile frequency and stimulates calcium current in cultured neonatal rat heart myocytes: insights into the underlying mechanisms. Circ. Res. 62:524-534.

31. Hori, M., K. Iwai, K. Iwakara, H. Sato, and A. Kiyabatake. 1989. Angiotensin II stimulates protein synthesis in neonatal rat cardiomyocytes through enhanced $\mathrm{Na}^{+} / \mathrm{H}^{+}$exchange (abstract). Circulation. 80(Suppl. II):450.

32. Eberli, F. R., C. S. Apstein, S. Ngoy, and B. H. Lorell. 1991. Specific inhibition of intrinsic renin-angiotensin system protects against exaggerated diastolic dysfunction of hypertrophied rat hearts during ischemia. Circ. Res. In press.

33. Freer, R. J., A. J. Pappano, M. R. Peach, K. T. Bing, M. J. McLean, S. Vogel, and N. Sperelakis. 1976. Mechanism for the positive inotropic effect of angiotensin II on isolated cardiac muscle. Circ. Res. 39:178-183.

34. Bonnardeaux, J. L., and D. Regoli. 1973. Action of angiotensin and analogues on the heart. Can. J. Physiol. Pharmacol. 52:50-60.

35. Kobayashi, M., Y. Furakawa, and S. Chiba. 1978. Positive chronotropic and inotropic effects of angiotensin II in the dog heart. Eur. J. Pharmacol. 50:1725.

36. Koch-Weser, J. 1965. Nature of the inotropic action of angiotensin on ventricular myocardium. Circ. Res. 16:230-237.

37. Baker, K. M., and H. A. Singer. 1988. Identification and characterization of guinea pig angiotensin II ventricular and atrial receptors: coupling to inositol phosphate production. Circ. Res. 62:896-904.

38. Cunningham, M. J., C. S. Apstein, E. O. Weinberg, W. M. Vogel, and B. H. Lorell. 1990. Influence of glucose and insulin on the exaggerated diastolic and systolic dysfunction of the hypertrophied rat heart during hypoxia. Circ. Res. 66:406-415.

39. Remme, W. J., H. A. C. M. Kruyssen, M. P. Look, M. Bootsma, and P. W. de Leeuw. 1990. Enalaprilat acutely reduces myocardial ischemia in normotensive patients with coronary artery disease through neurohumoral modulation. In Metabolic and Neurohumoral Aspects of Acute Myocardial Ischemia in Man. Volume 2. W. J. Remme, editor. CASPARIE (Publ.), Heerhugowaard. 346-362.

40. Rousseau, M. F., O. Gurne, C. Van Eyll, C. R. Benedict, and H. Pouleur. 1990. Effects of benazeprilat on left ventricular systolic and diastolic function and neurohumoral status in patients with ischemic heart disease. Circulation. 81(2 Suppl.):III123-III129.

41. Xiang, J., W. Linz, H. Becker, D. Ganten, R. E. Lang, B. Scholkens, and T. H. Unger. 1985. Effects of converting enzyme inhibitors: ramipril and enalapril on peptide action and sympathetic neurotransmission in the isolated heart. Eur. J. Pharmacol. 113:215-223.

42. Dosemeci, A., R. Dhallen, N. M. Cohen, W. J. Lederer, and T. B. Rogers. 1988. Phorbol ester increases calcium current and simulates the effects of angiotensin II on cultured neonatal rat heart myocytes. Circ. Res. 62:347-357.

43. Nosek, T. M., M. F. Williams, S. T. Aeigler, and R. E. Godt. 1986. Inosito triphosphate enhances calcium release in skinned cardiac and skeletal muscle. Am. J. Physiol. 250(Cell. Physiol. 19):C807-C811.

44. Nabika, T., P. A. Velletri, W. Lovenberg, and M. A. Beaven. 1985. Increase in cytosolic calcium and phosphoinositide metabolism induced by angiotensin II and [Arg] vasopressin in vascular smooth muscle cells. J. Biol. Chem. 260:4661-4670.

45. Gwathmey, J. K., and R. Hajjar. 1990. Effect of protein kinase C activation on sarcoplasmic reticulum function and apparent myofibrillar $\mathrm{Ca}^{2+}$ sensitivity in intact and skinned muscles from normal and diseased human myocardium. Circ. Res. 67:744-752.

46. Yuan, S., F. A. Sunahara, and A. K. Sen. 1987. Tumor-promoting phorbol esters inhibit cardiac functions and induce redistribution of protein kinase $C$ in perfused beating rat heart. Circ. Res. 61:372-378.

47. Moorman, J. R., G. E. Kirsch, A. E. Lacerda, and A. M. Brown. 1989. Angiotensin II modulates cardiac $\mathrm{Na}^{+}$channels in neonatal rat. Circ. Res. 65:1804-1809.

48. Nishizuka, Y. 1986. Studies and perspectives of protein kinase C. Science (Wash. DC). 233:305-312.

49. Lorell, B. H., E. O. Weinberg, H. Ikenouchi, and W. H. Barry. 1990. Change of Indo-1 $\left[\mathrm{Ca}^{2+}\right]_{i}$ transients and contractility induced by angiotensin II. Circulation. 82(Suppl. III): 141.

50. Kohmoto, O., K. W. Spitzer, M. A. Moveseian, and W. H. Barry. 1990. Effects of intracellular acidosis on $\left[\mathrm{Ca}^{2+}\right]_{\text {i }}$ transients, transsarcolemmal $\mathrm{Ca}^{2+}$ fluxes, and contraction in ventricular myocytes. Circ. Res. 66:622-623.

51. Urata, H., B. Healy, R. W. Stewart, F. M. Bumpus, and A. Husain. 1990. Angiotensin II-forming pathways in normal and failing human hearts. Circ. Res. 66:883-890.

52. Hirsch, A. T., C. E. Talsness, H. Schunkert, M. Paul, and V. J. Dzau. 1991. Tissue-specific activation of cardiac angiotensin converting enzyme in experimental heart failure. Circ. Res. 69:475-482. 